\title{
COMPRESSED SENSING OF EXCITATORY POSTSYNAPTIC POTENTIAL BIO-SIGNALS
}

\author{
Hyejin An and Hyun-Chool Shin \\ Department of Electronic Engineering, \\ Soongsil University, Seoul, Republic of Korea \\ $\{a h j$, shinhc\} @ssu.ac.kr
}

\begin{abstract}
To reduce the size of the biosignal data is important because a huge amount of data is made by various experiments. In the paper, we efficiently compress the excitatory postsynaptic potentials (EPSPs) which is one of the biosignal types. To the best of authors' knowledge, EPSPS compression has not been studied yet. The EPSP signal has a feature that the adjacent signals in single excitatory postsynaptic potential have similar characteristics. Using this feature, we propose a method which removes temporal redundancy and statistical redundancy of EPSPS. The compressed and reconstructed EPSPs are similar to the original signal without the loss of analytic information.
\end{abstract}

\section{KEYWORDS}

Excitatory Postsynaptic Potentials, Compressed Sensing, Long-term Potentiation, Long-term Depression, Data Compression

\section{INTRODUCTION}

Biosignals contain highly important information with which researchers in neuroscience and neural engineering fields attempt to disclose the neurological mechanisms of living organisms by measuring and analyzing various biosignals. Biosignals can be recorded using a variety of forms such as electroencephalogram (EEG), electrocorticography (ECoG), neural spike train, electrocardiography (ECG), electromyography (EMG), and excitatory postsynaptic potentials (EPSPs). To acquire more information about living organisms, researchers are recording biosignals by increasing the number of sensors, the measurement time, the number of experiment samples, and the measurement frequency. As a result, storage space, analysis time and data bandwidth for biosignals are being increasing as well. These problems can be solved with integrated memory chips, fast data processors and data communication technologies, but a more efficient method is to use data compression technology with which the biosignal data size is reduced while minimizing information loss.

Many data compression researches have been conducted to achieve the above-mentioned goals in recent years [1-5]. Sriraam and Eswaran [1] compressed EEG obtained from the scalp. Salman et al. [2] restored EMG using convex optimization to reduce energy consumption. Allstot et al. [3] 
conducted a study similar to [2] using ECG signals. In addition, Hegde et al. [4] proposed a compression method by creating a neuronal spike train model.

There are other studies about the compression of biosignals as well, but most studies have concentrated on the development of signal compression technology for EEG, ECoG, and neural spike trains acquired from in-vivo experiments. Although in-vitro or ex-vivo experiments are important in studying the neurological mechanism and biosignals recorded from these experiments require a large storage space [6-9], no study on the compression of EPSPs has been done. In general, EPSPs create data of $16 \mathrm{Kbits}(10,000 \mathrm{~Hz} \times 0.1 \mathrm{sec} \times 16 \mathrm{bits})$ in a single sensor with a single measurement. Assuming that 400 repetitive experiments using four to eight measurement sensors are conducted, the data recorded in a single experiment is approximately 26 51 Mbits, which is very large. In addition, experiments related to EPSPs measure biosignals through a large number of experiment samples with various parameters. As a result, actual data amounts could increase further.

This paper proposes a method that can compress EPSPs signals within a range that has no effect on the analysis of the EPSPs. The EPSPs signal occurs in synapses where two neurons are close to each other and it is created because the membrane potential of neurons behind the synapse increases due to neurotransmitters secreted from neurons in front of the synapse. Thus, changes in the EPSPs signal over time are slow and not large compared to neural spike trains or EEG. Inspired by this fact, EPSPs are compressed by eliminating information redundancy between adjacent signals.

This paper deals with the EPSPs signal which has not been tried for compression. The proposed method is suitable for the features of the EPSPs signal and includes uncomplicated arithmetic operations.

\section{MATERIALS AND METHODS}

\subsection{Materials}

EPSPs were measured via neurons of the hippocampus, which plays an important role in learning and memory. EPSPs signals were measured when hippocampus slices obtained from mice were stimulated. Normally, a single mouse has four to six slices, and a single slice can be a sensing channel. For both long-term potentiation (LTP) and long-term depression (LTD), experiments were conducted for 100 minutes, and the data for 80 minutes were used to draw a slope graph. A single EPSPs signal was measured every 15 seconds. The LTP and LTD experiments were conducted 381 times and 435 times, respectively. During the experiment, slices were immersed in an ACSF solution at approximately $28^{\circ} \mathrm{C}$ while bubbling was maintained as a mixed gas of $95 \%$ oxygen and $5 \%$ carbon dioxide was introduced at a rate of $4 \mathrm{ml} / \mathrm{min}$ for $\mathrm{pH}$ adjustment. The LTP was induced by providing theta burst stimulation (TBS) for one minute 40 minutes after the experiment started while LTD was induced by providing single-pulse low-frequency stimulation (SP-LFS) six times per minute for 15 minutes.

\subsection{Methods}

To compress the signals, it is necessary to eliminate temporal redundancy and statistical redundancy. In this paper, two signal-processing filters were used to eliminate temporal redundancy. The first filter was a low-pass filter (LPF), which passes only frequencies below 300 
$\mathrm{Hz}$, and the second filter was a first-order difference filter that calculated a difference between adjacent EPSPs signals. Since adjacent signals in single EPSPs change slowly and have similar characteristics, EPSPs after filtering become sparse.

Once temporal redundancy is eliminated, a compressed sensing technique was applied to eliminate statistical redundancy in spare EPSPs [10]. Assuming that the signal to be compressed is $\mathbf{x} \in \mathbb{R}^{N \times 1}$ and the compressed signal is $\mathbf{y} \in \mathbb{R}^{M \times 1}$, then it can be expressed as follows:

$$
\mathbf{y}=\boldsymbol{\Phi} \mathbf{x}
$$

where $\boldsymbol{\Phi} \in \mathbb{R}^{M \times N}$ is called a sensing matrix, which uses a binary matrix consisting of 0 and 1 in general. The most important point in compressed sensing is to express a signal to be sparse. To do this, conventionally, new sparse vector $\mathbf{z} \in \mathbb{R}^{N \times 1}$ and dictionary matrix $\mathbf{D} \in \mathbb{R}^{N \times N}$ are introduced so that $\mathbf{x}$ is expressed with $\mathbf{z}$ and $\mathbf{D}$, thereby generating sparse $\mathbf{x}$.. A matrix used in $\mathbf{D}$ is an inverse discrete cosine transform (DCT) or inverse discrete wavelet transform (DWT), in which $\mathbf{z}$ uses DCT or DWT coefficients. This procedure is quite complex. However, in this paper, we easily obtain sparse $\mathbf{x}$ by eliminating temporal redundancy without using conventional method. Since EPSPs show slow transition in time, the spare $\mathbf{x}$ is obtained by filtering EPSPs with a first-order difference filter.

Block sparse Bayesian learning-bound optimization (BSBL-BO) [11] was applied to restore $\hat{\mathbf{x}}$ from the compressed EPSPs signal $\mathbf{y}$. The compression ratio (CR) was calculated using

$$
\mathrm{CR}=\frac{\text { the number of bits in } \mathbf{x}}{\text { the number of bits in } \mathbf{y}} \text {. }
$$

A compression ratio of LTP and LTD has three values: 2, 3, and 4. The higher the compression ratio, the more often compression occurs.

To compare the similarity between restored and original signals, the normalized mean square error (NMSE) [12] was used.

$$
\operatorname{NMSE}=\frac{\|\mathbf{x}-\hat{\mathbf{x}}\|_{2}^{2}}{\|\mathbf{x}\|_{2}^{2}}
$$

where $\mathrm{x}$ is an original EPSPs signal and $\hat{\mathbf{x}}$ is a restored EPSPs signal. The closer the NMSE value is to 0 , the closer it is to the original signal.

\section{RESULTS}

Figure 1 shows the comparison of the original single EPSPs signal, $\mathbf{x}$ and the restored single EPSPs signal, $\hat{\mathbf{x}}$ for LTP and LTD. In the figure, a compression ratio was set to 2; a dotted line represents the original EPSPs signal while a solid line represents the recovered EPSPs signal. Figure 2 shows the comparison between the original EPSPs and the restored signals, depicting NMSE compared over various compression ratios. For LTP, depending on compression ratios 2, 3 , and 4 , the mean and standard deviation (STD) of the NMSE were $0.020 \pm 0.014,0.15 \pm 0.032$ and $0.35 \pm 0.060$, respectively. For LTD, depending on the compression ratio, the mean and STD were $0.084 \pm 0.021,0.18 \pm 0.021$ and $0.46 \pm 0.026$, respectively. As the compression ratio becomes lower, which means more compression, the NMSE increases, which means the restoration performance decreases. 

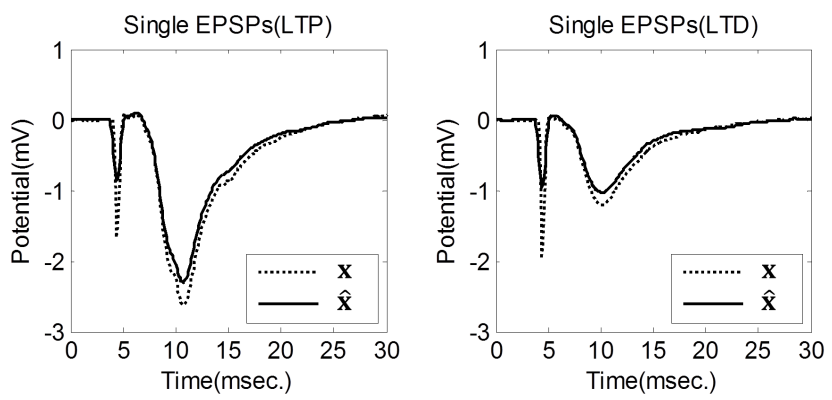

Figure 1. Comparison of original signal and recovered signal. They are the single EPSPs signals generated after the stimulation $(\mathrm{CR}=2)$.
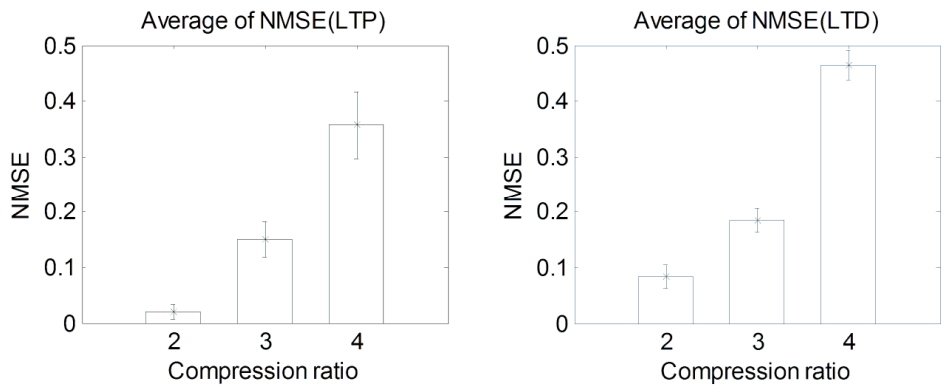

Figure 2. NMSE mean and STD comparison according to compression ratio. NMSE compares the difference between the original signal and the recovered signal.

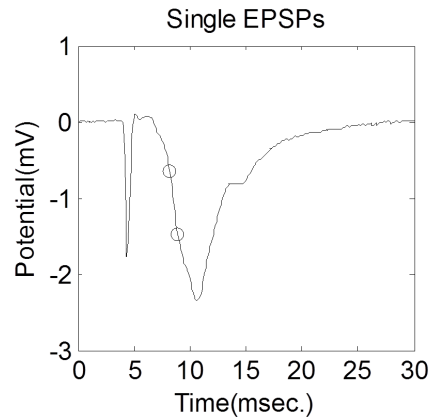

Figure 3. Single EPSPs signal in which a section slope is calculated. The two points in the middle are a $1 / 3 \sim 2 / 3$ section designated for the slope calculation.

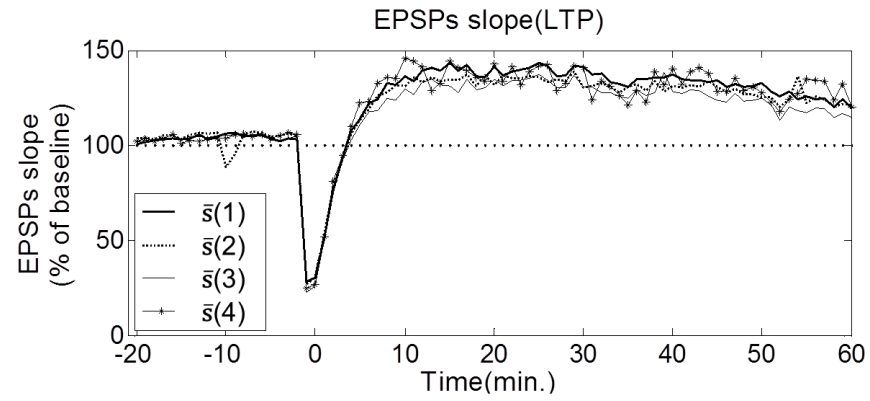




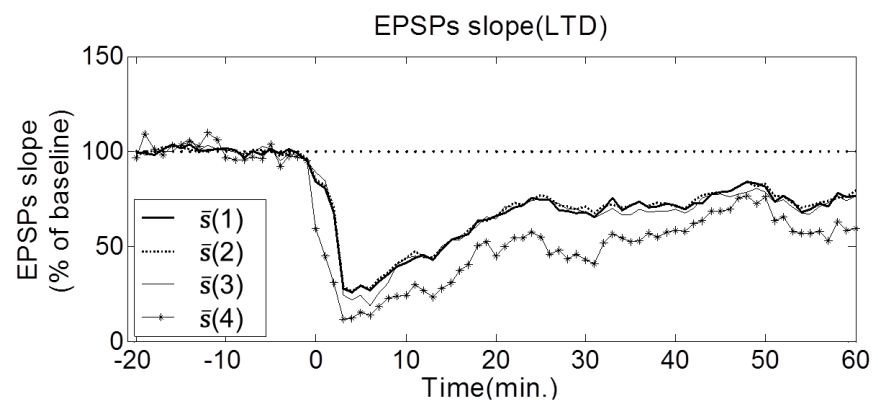

Figure 4. Comparison of changes in slopes calculated from original single EPSPs signals and recovered single EPSPs signals. On the time axis in the figure, 0 refers to a time when the stimulation was given and the slope change over time from 20 minutes prior to the stimulation and 60 minutes after the stimulation, which was a total of 80 minutes, shown as a percentage. Each point represents a slope mean of EPSPs signals calculated every one minute. The values in the brackets refer to compression ratios. $\overline{\mathbf{s}}(1)$ means the slope without compression.

To analyze EPSPs signals in general, the slope in temporal EPSPs is compared before and after the stimulation is given. For the first step in the comparison, a slope was calculated as a section of $1 / 3 \sim 2 / 3$ set in the single EPSPs signal slope, as shown in Figure 3. Each single EPSPs signal has its own single calculated slope. A method of calculating a slope is to divide changes in potential in the designated section by time. As a next step, a mean is calculated every one minute using a single EPSPs signal slope in the section from 20 minutes prior to stimulation to 60 minutes after stimulation. The baseline that is a criterion for comparison before and after stimulation is a slope mean of the EPSPs signal calculated from 20 minutes prior to stimulation until the stimulation was given; this value was again converted into $100 \%$ proportion. All slope means obtained every minute are normalized using the above mentioned criterion, and the slope, $\bar{s}(t)$ calculated by

$$
\bar{s}(t)=\frac{s(t)}{\frac{1}{N} \sum s_{b}(t)} \times 100
$$

where $s(t)$ is the EPSPs slope whose means are obtained every one minute. $s_{b}(t)$ is a part of $s(t)$ that corresponds to the baseline section which is for 20 minutes prior to stimulation. $\frac{1}{N} \sum s_{b}(t)$ is the mean of $s_{b}(t)$. This $\bar{s}(t)$ is the normalized slope value of $s(t)$.

Figure 4 shows the normalized slope, $\bar{s}(t)$ from the original EPSPs and the recovered ones. For the low compression ratio, the figure shows that the slope graph is very similar to the slope of the original signal. When the LTD is restored with a compression ratio of 4 , the slope value seems to change more than the slope of the original signal, but it did not affect the overall slope trend significantly.

\section{CONCLUSION}

EPSPs signals have been studied by many researchers in recent years and require a large storage capacity whenever experiments are conducted. In addition, researchers set various parameters to conduct experiments. Because of this, it is very important to reduce the data size of EPSPs signals. However, unlike other biosignals, no studies have been done on EPSPs compression. In this study, an efficient compression method was proposed without any problems in analyzing EPSPs. Using the proposed method, EPSPs signals can be stored and analyzed with a smaller amount of data than that of the original signals. 


\section{ACKNOWLEDGEMENTS}

This research was supported by the Original Technology Research Program for Brain Science through the National Research Foundation of Korea (NRF) funded by the Ministry of Education, Science and Technology (NRF-2011-0019212).

\section{REFERENCES}

[1] Sriraam, N. \& Eswaran, C., (2004) "EEG signal compression using radial basis neural networks", Journal of Mechanics in Medicine and Biology, Vol. 4, No. 2, pp143-152.

[2] Salman, A., Allstot, E.G., Chen, A.Y., Dixon, A.M.R., Gangopadhyay, D. \& Allstot, D.J., (2011) "Compressive sampling of EMG bio-signals", IEEE International Symposium on Circuits and Systems, Rio de Janeiro, Brazil, pp2095-2098.

[3] Allstot, E.G., Chen, A.Y., Dixon, A.M.R., Gangopadhyay, D. \& Allsot, D.J., (2010) "Compressive sampling of ECG bio-signals: Quantization noise and sparsity considerations", IEEE Conference on Biomedical Circuits and Systems Conference, Paphos, Cyprus, pp41-44.

[4] Hegde, C., Duarte, M.F. \& Cevher, V., (2009) "Compressive sensing recovery of spike trains using a structured sparsity model", Signal Processing with Adaptive Sparse Structured Representations, Saint Malo, France.

[5] Sharma, L.N., Dandapat, S. \& Mahanta, A., (2012) "Multichannel ECG data compression based on multiscale principal component analysis", IEEE Transactions on Information Technology in Biomedicine, Vol. 16, No. 4, pp730-736.

[6] Womble, M.D., Andrew, J.A. \& Crook, J.J., (2002) "17ß-Estradiol reduces excitatory postsynaptic potential (EPSP) amplitude in rat basolateral amygdala neurons", Neuroscience letters, Vol. 331, No. 2, pp83-86.

[7] Choi, T.-Y., Kwon, J.E., Durrance, E.S., Jo, S.-H., Choi, S.-Y. \& Kim, K.-T., (2014) "Melatonin inhibits voltage-sensitive $\mathrm{Ca} 2+$ channel-mediated neurotransmitter release", Brain Research, Vol. 1557, pp34-42.

[8] Seo, J., Kim, K., Jang, S., Han, S., Choi, S.-Y., \& Kim, E., (2012) "Regulation of hippocampal longterm potentiation and long-term depression by diacylglycerol kinase $\zeta$ ", Hippocampus, Vol. 22, No. 5, pp1018-1026.

[9] Bliss, T.V.P. \& Cooke, S.F., (2011) "Long-term potentiation and long-term depression: a clinical perspective", Clinics, Vol. 66, pp3-17.

[10] Candès, E.J. \& Wakin, M.B., (2008) "An introduction to compressive sampling", IEEE Transactions on Signal Processing Magazine, Vol. 25, No. 2, pp21-30.

[11] Zhang, Z. \& Rao, B.D., (2013) "Extension of SBL algorithms for the recovery of block sparse signals with intra-block correlation”, IEEE Transactions on Signal Processing, Vol. 61, No.8, pp2009-2015.

[12] Zhang, Z., Jung, T.-P., Makeig, S. \& Rao, B.D., (2013) "Compressed sensing for energy-efficient wireless telemonitoring of noninvasive fetal ECG via block sparse Bayesian learning", IEEE Transactions on Biomedical Engineering, Vol. 60, No. 2, pp300-309. 


\section{AUTHORS}

Hyejin An was born in Seoul, Korea, in 1988. She received the B.Sc. degrees in electronic engineering from Soongsil University, Korea, in 2012, where she is currently working toward the Ph.D. degree in signal processing in the Department of Electronic Engineering. Her research interests include neural and intelligent signal processing focusing on brain and bio-signals.

Hyun-Chool Shin (M'04) was born in Seoul, Korea, on June 21, 1974. He received the B.Sc., M.Sc., and Ph.D. degrees in electronic and electrical engineering from Pohang University of Science and Technology (POSTECH), Korea, in 1997, 1999, and 2004 respectively. From 2004 to 2007, he has been a postdoctoral researcher at the Department of Biomedical Engineering, Johns Hopkins University, School of Medicine. Since 2007, he is currently an associate professor of electronic engineering at the Soongsil University. His research interests include neural and intelligent signal processing focusing on brain and bio-signals and radar array signals.
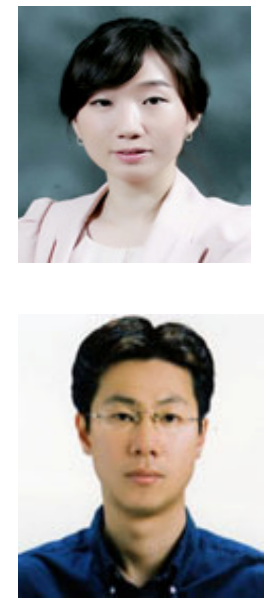